\title{
Global attractor for the Navier-Stokes equations in a cylindrical pipe
}

\author{
by Piotr KacPRZYK (Warszawa)
}

\begin{abstract}
Global existence of regular special solutions to the Navier-Stokes equations describing the motion of an incompressible viscous fluid in a cylindrical pipe has already been shown. In this paper we prove the existence of the global attractor for the Navier-Stokes equations and convergence of the solution to a stationary solution.
\end{abstract}

1. Introduction. We consider viscous incompressible fluid motions in a finite cylinder with large inflow and outflow and under boundary slip conditions. The following initial-boundary value problem is examined:

$$
\begin{array}{ll}
v_{, t}+v \cdot \nabla v-\operatorname{div} \mathbb{T}(v, p)=f & \text { in } \Omega^{T}=\Omega \times(0, T), \\
\operatorname{div} v=0 & \text { in } \Omega^{T}, \\
v \cdot \bar{n}=0 & \text { on } S_{1}^{T}=S_{1} \times(0, T), \\
\nu \bar{n} \cdot \mathbb{D}(v) \cdot \bar{\tau}_{\alpha}+\gamma v \cdot \bar{\tau}_{\alpha}=0, \quad \alpha=1,2, & \text { on } S_{1}^{T}, \\
v \cdot \bar{n}=d & \text { on } S_{2}^{T}=S_{2} \times(0, T), \\
\bar{n} \cdot \mathbb{D}(v) \cdot \bar{\tau}_{\alpha}=0, \quad \alpha=1,2, & \text { on } S_{2}^{T}, \\
\left.v\right|_{t=0}=v(0) & \text { in } \Omega, \\
\int_{\Omega} p d x=0, &
\end{array}
$$

where $\Omega \subset \mathbb{R}^{3}, S=S_{1} \cup S_{2}=\partial \Omega, v=v(x, t)=\left(v_{1}(x, t), v_{2}(x, t), v_{3}(x, t)\right) \in$ $\mathbb{R}^{3}$ is the velocity vector of the fluid motion, $p=p(x, t) \in \mathbb{R}^{1}$ the pressure, $f=f(x, t)=\left(f_{1}(x, t), f_{2}(x, t), f_{3}(x, t)\right) \in \mathbb{R}^{3}$ the external force field, $\bar{n}$ the unit outward vector normal to the boundary $S$, and $\bar{\tau}_{\alpha}, \alpha=1,2$, are tangent vectors to $S$. Moreover, $\mathbb{T}(v, p)$ is the stress tensor of the form

$$
\mathbb{T}(v, p)=\nu \mathbb{D}(v)-p I,
$$

2010 Mathematics Subject Classification: 35Q35, 76D03, 76D05.

Key words and phrases: Navier-Stokes equations, inflow-outflow problem, slip boundary conditions, cylindrical domains, global existence of regular solutions. 
where $\nu$ is the constant viscosity coefficient, $I$ the unit matrix and $\mathbb{D}(v)$ the dilatation tensor,

$$
\mathbb{D}(v)=\left\{v_{i, x_{j}}+v_{j, x_{i}}\right\}_{i, j=1,2,3} .
$$

Finally, $\gamma>0$ is the slip coefficient.

By $\Omega \subset \mathbb{R}^{3}$ we denote a cylindrical type domain parallel to the $x_{3}$ axis with arbitrary cross section. We assume that $S_{1}$ is the part of the boundary which is parallel to the $x_{3}$ axis and $S_{2}$ is perpendicular to $x_{3}$. Hence

$$
\begin{aligned}
S_{1} & =\left\{x \in \mathbb{R}^{3}: \varphi\left(x_{1}, x_{2}\right)=c_{0},-a<x_{3}<a\right\}, \\
S_{2}(-a) & =\left\{x \in \mathbb{R}^{3}: \varphi\left(x_{1}, x_{2}\right)<c_{0}, x_{3}=-a\right\}, \\
S_{2}(a) & =\left\{x \in \mathbb{R}^{3}: \varphi\left(x_{1}, x_{2}\right)<c_{0}, x_{3}=a\right\},
\end{aligned}
$$

where $a, c_{0}$ are given positive numbers and $\varphi\left(x_{1}, x_{2}\right)=c_{0}$ describes a sufficiently smooth closed curve in the plane $x_{3}=$ const.

To describe inflow and outflow we define

$$
d_{1}=-\left.v \cdot \bar{n}\right|_{S_{2}(-a)}, \quad d_{2}=\left.v \cdot \bar{n}\right|_{S_{2}(a)},
$$

so $d_{i} \geq 0, i=1,2$, and by $(1.1)_{2,3}$ and (1.2) we have the compatibility condition

$$
\Phi \equiv \int_{S_{2}(-a)} d_{1} d S_{2}=\int_{S_{2}(a)} d_{2} d S_{2}
$$

where $\Phi$ is the flux.

Let us introduce an extension $\alpha=\alpha(x, t) \in \mathbb{R}$ such that

$$
\left.\alpha\right|_{S_{2}(-a)}=d_{1},\left.\quad \alpha\right|_{S_{2}(a)}=d_{2} .
$$

Then equations (1.1) $)_{2,3,6}$ and (1.3) imply the compatibility condition

$$
\int_{\Omega} \alpha_{, x_{3}} d x=-\left.\int_{S_{2}(-a)} \alpha\right|_{x_{3}=-a} d S_{2}+\left.\int_{S_{2}(a)} \alpha\right|_{x_{3}=a} d S_{2}=0 .
$$

In [14, 15, 16] the long time existence of solutions is proved in non-axially cylindrical domains. In [17] the existence is proved in Besov spaces and in [2, 9] the proof of existence is simplified to use Sobolev spaces only. In [8] the global existence of solution is proved by prolongation of long time solutions from [9].

Now we explain why the global existence is proved in the two steps. We can prove global existence directly but then we have to assume that the time integral norms of data functions are finite on $(0, \infty)$ (see the norms before Theorem 2.1).

This means that all data functions must vanish sufficiently fast as $t \rightarrow \infty$. To omit these restrictions we prove the existence of solutions on the interval $(0, T)$ with fixed $T$, without smallness restriction on $T$. Then to prove global existence we repeat the considerations from the interval $(0, T)$ on all intervals 
$(k T,(k+1) T), k \in \mathbb{N}$. For this purpose we need to know that the norms of data at time $t=k T$ are not greater than the same norms at $t=0$.

This can be shown by using the natural decay properties for solutions of the Navier-Stokes equations and $T$ sufficiently large. In this way the initial data at $t=k T$ are not greater than the initial data at $t=0$. In [17] the inflow-outflow problem is considered, and in [3] global existence is proved by prolongation of long time solutions from [2].

This paper extends the result from [7] to the inflow-outflow case. This extension is difficult because the inflow-outflow flux makes the problems considered in this paper nonhomogeneous. This implies that solvability of such a problem must be shown in a different way than in [7]. Moreover, many new expressions connected with the inflow-outflow flux must be estimated. Hence, we show the existence of a global attractor for the Navier-Stokes equations in cylindrical domains in the inflow-outflow case.

Now we formulate the main result. Let $\delta>0$ be fixed and

$$
\bar{V}=\left\{v \in C^{\infty}(\Omega): \operatorname{div} v=0,\left.v \cdot \bar{n}\right|_{S_{1}}=0,\left.v \cdot \bar{n}\right|_{S_{2}}=d,\left|v_{x_{3}}\right|_{2, \Omega}<\delta\right\},
$$
then

$$
\begin{aligned}
H & \equiv \text { closure of } \bar{V} \text { in the } L_{2} \text {-norm, } \\
V & \equiv \text { closure of } \bar{V} \text { in the } H^{1} \text {-norm. }
\end{aligned}
$$

Let $v(t)$ be a weak global solution to problem (1.1) with initial data $v_{0}=$ $v(0)$. Then $S(t): H \rightarrow H$ is a semiprocess defined by $v(t)=S(t) v(0)$.

THEOREM 1.1. There exists a global attractor $\mathcal{A}$ in $V$ for the semiprocess $\{S(t)\}_{t \geq 0}$ (see also (3.1)). The attractor is bounded in $H^{1}(\Omega)$ and compact and connected in $V$. It attracts bounded sets in $V$.

2. Notation and auxiliary results. To simplify the presentation we introduce the following notation:

$$
\begin{array}{ll}
|u|_{p, Q}=\|u\|_{L_{p}(Q)}, & Q \in\left\{\Omega^{T}, S^{T}, \Omega, S\right\}, p \in[1, \infty], \\
\|u\|_{s, Q}=\|u\|_{H^{s}(Q)}, & Q \in\{\Omega, S\}, s \in \mathbb{R}_{+} \cup\{0\}, \\
\|u\|_{s, Q^{T}}=\|u\|_{W_{2}^{s, s / 2}\left(Q^{T}\right)}, & Q \in\{\Omega, S\}, s \in \mathbb{R}_{+} \cup\{0\}, \\
|u|_{p, q, Q^{T}}=\|u\|_{L_{q}\left(0, T ; L_{p}(Q)\right)}, & Q \in\{\Omega, S\}, p, q \in[1, \infty], \\
\|u\|_{s, q, Q^{T}}=\|u\|_{W_{q}^{s, s / 2}\left(Q^{T}\right)}, & Q \in\{\Omega, S\}, s \in \mathbb{R}_{+} \cup\{0\}, q \in[1, \infty], \\
\|u\|_{s, q, Q}=\|u\|_{W_{q}^{s}(Q)}, & Q \in\{\Omega, S\}, s \in \mathbb{R}_{+} \cup\{0\}, q \in[1, \infty] .
\end{array}
$$

By $c$ we denote a generic constant which changes its magnitude from formula to formula. By $\bar{c}(\sigma), \varphi(\sigma)$ we understand generic functions which are always positive and increasing. Finally, we do not distinguish the scalarvalued and vector-valued functions. 
We introduce the space

$$
\begin{aligned}
V_{2}^{k}\left(\Omega^{T}\right)=\left\{u:\|u\|_{V_{2}^{k}\left(\Omega^{T}\right)}=\underset{t \in(0, T)}{\operatorname{ess} \sup }\|u\|_{H^{k}(\Omega)}\right. & \\
& \left.+\left(\int_{0}^{T}\|\nabla u(t)\|_{H^{k}(\Omega)}^{2} d t\right)^{1 / 2}<\infty\right\}, \quad k \in \mathbb{N} .
\end{aligned}
$$

Finally, we introduce the quantities

$$
\begin{array}{ll}
h=v_{x_{3}}, & q=p_{, x_{3}}, \quad g=f_{, x_{3}}, \\
w=v_{3}, & \chi=v_{2, x_{1}}-v_{1, x_{2}} .
\end{array}
$$

Lemma 2.1 (Korn inequality; see [14]). Assume that $|\mathbb{D}(v)|_{2, \Omega}^{2}<\infty$, $\left.v \cdot \bar{n}\right|_{S}=0, \operatorname{div} v=0$. If $\Omega$ is not axially symmetric, then there exists a constant $c>0$ such that

$$
\|v\|_{1, \Omega}^{2} \leq c|\mathbb{D}(v)|_{2, \Omega}^{2} .
$$

First we need the estimates and the uniform Gronwall inequality.

Lemma 2.2. The solution $v \in H^{2}(\Omega)$ of the elliptic problem

$$
\begin{aligned}
& \operatorname{div} \mathbb{D}(v)=f \\
& \left.v \cdot \bar{n}\right|_{S_{1}}=0 \\
& \left.v \cdot \bar{n}\right|_{S_{2}}=d \\
& \left(\bar{n} \cdot \mathbb{D}(v) \cdot \bar{\tau}_{\alpha}+v \cdot \bar{\tau}_{\alpha}\right)_{\left.\right|_{S_{1}}}=0, \quad \alpha=1,2 \\
& \left.\bar{n} \cdot \mathbb{D}(v) \cdot \bar{\tau}_{\alpha}\right|_{S_{2}}=0, \quad \alpha=1,2
\end{aligned}
$$

satisfies the estimate

$$
\|v\|_{2, \Omega} \leq c\left(|f|_{2, \Omega}+|v|_{2, \Omega}+\|d\|_{3 / 2,2, S_{2}}+\left\|v \cdot \tau_{\alpha}\right\|_{1 / 2,2, S_{1}}\right) .
$$

Lemma 2.3. The solution $(v, p) \in H^{2}(\Omega) \times H^{1}(\Omega)$ of the elliptic problem

$$
\begin{aligned}
& \operatorname{div} \mathbb{T}(v, p)=f \\
& \operatorname{div} v=0 \\
& \left.v \cdot \bar{n}\right|_{S_{1}}=0 \\
& \left.v \cdot \bar{n}\right|_{S_{2}}=d \\
& \left(\bar{n} \cdot \mathbb{D}(v) \cdot \bar{\tau}_{\alpha}+v \cdot \bar{\tau}_{\alpha}\right)_{S_{1}}=0, \quad \alpha=1,2, \\
& \left.\bar{n} \cdot \mathbb{D}(v) \cdot \bar{\tau}_{\alpha}\right|_{S_{2}}=0, \quad \alpha=1,2,
\end{aligned}
$$

satisfies the estimate

(2.5) $\|v\|_{2, \Omega}+|\nabla p|_{2, \Omega} \leq c\left(|f|_{2, \Omega}+|v|_{2, \Omega}+\|d\|_{3 / 2,2, S_{2}}+\left\|v \cdot \tau_{\alpha}\right\|_{1 / 2,2, S_{1}}\right)$.

Lemmas 2.2, 2.3 follow from the theory of boundary value problems for Douglis-Nirenberg elliptic systems (see [10]). 
Lemma 2.4 (uniform Gronwall inequality; see [12, Ch. 3, Lemma 1.1]). Let $g, h, y:\left[t_{0}, \infty\right) \rightarrow(0, \infty)$ be continuous functions. Assume that for some $r>0$ and all $t>t_{0}$ the inequalities

$$
\begin{aligned}
& y^{\prime}(t) \leq g(t) y(t)+h(t), \\
& \int_{t}^{t+r} g(s) d s \leq a_{1}, \quad \int_{t}^{t+r} h(s) d s \leq a_{2}, \quad \int_{t}^{t+r} y(s) d s \leq a_{3}
\end{aligned}
$$

hold. Then $y$ satisfies the uniform estimate

$$
y(t+r) \leq\left(\frac{a_{3}}{r}+a_{2}\right) e^{a_{1}} \quad \text { for } t>t_{0} .
$$

The existence of regular solutions to the problem (1.1) has been proved in [2] by the Leray-Schauder fixed point theorem assuming smallness of the $L_{2}$-norms of the derivative, with respect to the variable along the axis of the cylinder, of the external force and the initial velocity. Moreover, we need the density of the flux to have sufficiently small derivatives with respect to variables on $S_{2}$ and time. The next step is to obtain global in time solutions. This is shown in [3].

To formulate the main result from [3] we need the notation

$$
\begin{gathered}
\Gamma^{2}(t)=|\alpha|_{2, S_{1}}^{2}+|\alpha, t|_{6 / 5, \Omega}^{2}+\left|\alpha_{, x_{3} t}\right|_{6 / 5, \Omega}^{2} \\
+\left(1+\|\alpha\|_{1,3, \Omega}^{2}\right)|\nabla \alpha|_{2, \Omega}^{2}+|f|_{6 / 5, \Omega}^{2}, \\
\Gamma_{1}^{2}(k T, t)=\|\alpha\|_{L_{\infty}\left(k T, t ; L_{2}(\Omega)\right)}^{2}+\left\|\alpha_{, x_{3}}\right\|_{L_{\infty}\left(k T, t ; L_{2}(\Omega)\right)}^{2} \\
+\int_{k T}^{t}\left\|\alpha\left(t^{\prime}\right)\right\|_{1,2, \Omega}^{2} d t^{\prime}, \\
l_{1}^{2}(k T, t)=c \exp c\left(\left|d_{1}\right|_{3,6, S_{2} \times(k T, t)}^{6}+|\nabla \alpha|_{3,2, \Omega \times(k T, t)}^{2}\right) \\
\quad \cdot\left(\int_{k T}^{t} \Gamma^{2}\left(t^{\prime}\right) d t^{\prime}+\Gamma_{1}^{2}(k T, t)+|v(k T)|_{2, \Omega}^{2}\right), \\
G(k T, t)=l_{1}(k T, t)+\left\|d_{1}\right\|_{3 / 2,2, S_{2} \times(k T, t)}+|f|_{2, \Omega \times(k T, t)}, \quad F_{3}=(\operatorname{rot} v)_{3}, \\
+\left|F_{3}\right|_{10 / 7, \Omega \times(k T, t)}+\left|d_{1}\right|_{\infty, \Omega \times(k T, t)}, \quad \text { where } \\
G^{\prime}(k T, t)=|g|_{2, \Omega \times(k T, t)}+l_{1}(k T, t)+\left\|d_{1, x^{\prime}}\right\|_{3 / 2,2, S_{2} \times(k T, t)},
\end{gathered}
$$

where $g=f_{, x_{3}}$,

$$
\begin{aligned}
\eta(k T, t)= & \left\|d_{1, x^{\prime}}\right\|_{L_{\infty}\left(k T, t ; H^{1}\left(S_{2}\right)\right)}+\left\|d_{1, t}\right\|_{L_{2}\left(k T, t ; H^{1}\left(S_{2}\right)\right)} \\
& +\left\|f_{3}\right\|_{L_{2}\left(k T, t ; L_{4 / 3}(\Omega)\right)}+\|g\|_{L_{2}\left(k T, t ; L_{6 / 5}(\Omega)\right)}+\frac{1}{T} l_{1}(k T, t),
\end{aligned}
$$

where $t \in(k T,(k+1) T)$. 
Theorem 2.1 (global existence). Assume that $t \in(k T,(k+1) T), k \in$ $\mathbb{N} \cup\{0\} \equiv \mathbb{N}_{0}$,

$$
\begin{aligned}
& \int_{k T}^{t} \Gamma^{2}\left(t^{\prime}\right) d t^{\prime} \leq \int_{0}^{T} \Gamma^{2}\left(t^{\prime}\right) d t^{\prime} \\
& \Gamma_{1}(k T, t) \leq \Gamma_{1}(0, T), \quad G(k T, t) \leq G(0, T), \quad G^{\prime}(k T, t) \leq G^{\prime}(0, T), \\
& l_{1}(k T, t) \leq l_{1}(0, T), \quad \eta(k T, t) \leq \eta(0, T) .
\end{aligned}
$$

Assume that $\eta(k T,(k+1) T)$ is so small that there exists a positive constant A such that

$$
\varphi(A, G(k T, t)) \eta(k T, t)+G^{\prime}(k T, t)<A, \quad t \in(k T,(k+1) T],
$$

where $\varphi$ is some positive increasing function. Then there exists a solution to (1.1) such that

$$
\begin{aligned}
& \left\|v_{, x_{3}}\right\|_{W_{2}^{2,1}((k T,(k+1) T) \times \Omega)} \leq A, \\
& \|v\|_{W_{2}^{2,1}((k T,(k+1) T) \times \Omega)} \leq c\left(A^{2}+1\right),
\end{aligned}
$$

where $A>0$ is a constant chosen for a given $T$ and independent of $k \in \mathbb{N}$.

REMARK 2.1. We have to underline that the constant $A$ appearing in (2.7) need not be small so also the solution $(v, p)$ to problem (1.1) may not be small either (see (2.8)). However, to guarantee the existence of a constant $A$ satisfying $(2.7)$ we need sufficient smallness of the quantity $\eta(k T, t)$, $t \in(k T,(k+1) T)$. To satisfy this restriction we need smallness of $d_{, t}, d_{, x^{\prime}}$, $f_{3}, g$ in the norms in the definition of $\eta$. Moreover, we need $(1 / T) l_{1}(k T, t)$ to be sufficiently small. To guarantee this we require that $d_{1}(t)$ and $\alpha(t)$ decrease with time sufficiently fast and also $T$ is chosen sufficiently large. The above restrictions do not impose restrictions on the magnitudes of the initial velocity $v(0)$ and the external force $f$. Therefore $v, p$ are not small so the problem considered is not a problem with small data.

In this paper we will show the existence of the global attractor for problem (1.1). We will apply methods from [11] and use the theory of semiprocesses since in our case the external force $f$ depends on time.

3. Existence of the global attractor. In this section we prove the existence of the global attractor to the problem (1.1). We start by recalling some facts and definitions from [1, Ch. 4].

Let us rewrite equation $(1.1)_{1}$ in the abstract form

$$
v_{, t}=A(v, t)=A_{\sigma(t)}(v), \quad t \in \mathbb{R}^{+},
$$

where the right-hand side depends explicitly on the time symbol $\sigma(t)$, which is the collection of all time-dependent coefficients of the equation (in the Navier-Stokes equations that will be the time-dependent external forces). 
By $\Phi$ we denote some metric or Banach space which contains the values of $\sigma(t)$ for a.e. $t \in \mathbb{R}_{+}$. Moreover, we assume that $\sigma(t)$, as a function of $t$, belongs to a topological space $\Xi:=\left\{\xi(t), t \in \mathbb{R}_{+}: \xi(t) \in \Psi\right.$ for a.e. $\left.t \in \mathbb{R}_{+}\right\}$.

Replacing the symbol $\sigma(t)$ by the shifted symbol $\sigma(t+h)$ should not change the attractor, hence we introduce a translation invariant subspace $\Sigma \subseteq \Xi$ called the symbol space. Translation invariance means that for all $\sigma(t) \in \Sigma$ the relation $T(h) \sigma(t)=\sigma(t+h) \in \Sigma$ holds, where $T(h): \Xi \rightarrow \Xi$ is the shift operator. In our case, it will be convenient to set $\Sigma=\Sigma\left(\sigma_{0}\right) \equiv$ $\left\{\sigma_{0}(t+h): h \in \mathbb{R}^{+}\right\}$, where $\sigma_{0}$ is the time symbol of the initial equation and the closure is taken in the topology of $\Xi$.

Let $v(t)$ be a weak and global solution of problem (1.1) with initial data $v_{0}=v(0)$. We define a family of semiprocesses $\left\{U_{\sigma}(t, \tau)\right\}_{t \geq \tau \geq 0}$ acting on $H, U(t, \tau): H \rightarrow H$ by the formula

$$
v(t)=U_{\sigma}(t, \tau) v(\tau),
$$

where $v(\tau)$ is the initial condition and $\Sigma \ni \sigma(t)=f(\cdot, t)$ is the external force.

By $\mathcal{B}(H)$ we denote the family of all bounded sets of $H$.

Definition 3.1 (see [1, Ch. 4, Definition 3.2]). A family of semiprocesses $\left\{u_{\sigma}(t, \tau)\right\}_{t \geq \tau \geq 0}, \sigma \in \Sigma$, is said to be uniformly bounded if for any $B \in \mathcal{B}(H)$ we have

$$
\bigcup_{\sigma \in \Sigma} \bigcup_{\tau \in \mathbb{R}^{+}} \bigcup_{t \geq \tau} U_{\sigma}(t, \tau) B \in \mathcal{B}(H) .
$$

Definition 3.2 (see [1, Ch. 4, Definition 3.3]). A set $B_{0} \in H$ is said to be uniformly absorbing for the family of semiprocesses $\left\{U_{\sigma}(t, \tau)\right\}_{t \geq \tau \geq 0}$, $\sigma \in \Sigma$, if for any $\tau \in \mathbb{R}^{+}$and for every $B \in \mathcal{B}(H)$ there exists $t_{0}=t_{0}(\tau, B)$ such that $\bigcup_{\sigma \in \Sigma} U_{\sigma}(t, \tau) B \subseteq B_{0}$ for all $t \geq t_{0}$. If the set $B_{0}$ is compact, we call the family of semiprocesses uniformly compact.

Definition 3.3 (see [1, Ch. 4, Definition 3.4]). A set $P$ belonging to $H$ is called uniformly attracting for the family of semiprocesses $\left\{U_{\sigma}(t, \tau)\right\}_{t \geq \tau \geq 0}$, $\sigma \in \Sigma$, if for an arbitrary fixed $\tau \in \mathbb{R}^{+}$,

$$
\lim _{t \rightarrow \infty}\left(\sup _{\sigma \in \Sigma} \operatorname{dist}_{E}\left(U_{\sigma}(t, \tau) B, P\right)\right)=0 .
$$

If the set $P$ is compact, we call the family of semiprocesses uniformly asymptotically compact.

Definition 3.4 (see [1, Ch. 4, Definition 3.5]). A closed set $\mathcal{A}_{\Sigma} \subset H$ is called the uniform attractor of the family of semiprocesses $\left\{U_{\sigma}(t, \tau)\right\}_{t \geq \tau \geq 0}$, $\sigma \in \Sigma$, if it is uniformly attracting and contained in any closed uniformly attracting set of the family $\left\{U_{\sigma}(t, \tau)\right\}_{t \geq \tau \geq 0}, \sigma \in \Sigma$. 
The existence of the global attractor follows from the following theorem:

Theorem 3.1 (see [1, Ch. 4, Theorem 3.1]). If a family of semiprocesses $\left\{U_{\sigma}(t, \tau)\right\}_{t \geq \tau \geq 0}, \sigma \in \Sigma$, is uniformly asymptotically compact, then it possesses a uniform global attractor $\mathcal{A}_{\Sigma}$. The set $\mathcal{A}_{\Sigma}$ is compact in $H$.

The main result in this section reads.

THEOREM 3.2. There exists a global attractor $\mathcal{A}_{\Sigma}$ in $H$ for the family of semiprocesses $\left\{U_{\sigma}(t, \tau)\right\}_{t \geq \tau \geq 0}, \sigma \in \Sigma$, defined by (3.1). The attractor is bounded in $V$ and compact and connected in $H$. It attracts bounded sets in $H$.

To prove this theorem we need some estimates.

Lemma 3.1. There exists a bounded and absorbing set in $H$ for the family of semiprocesses $\left\{U_{\sigma}(t, \tau)\right\}_{t \geq \tau \geq 0}, \sigma \in \Sigma$.

Proof. In view of Theorem 2.1 we see that

$$
\limsup _{t \rightarrow \infty}\|v(t)\|_{L_{2}(\Omega)} \leq c\left(A^{2}+1\right) .
$$

Hence for every $v_{0} \in H$ there exists $t_{0}>0$ such that

$$
v(t) \in B\left(0, \rho_{1}\right) \quad \text { for all } t \geq t_{0},
$$

where $B\left(0, \rho_{1}\right)$ is the ball in $H$ centered at 0 with radius $\rho_{1}>c\left(A^{2}+1\right)$. If $B(0, r) \subset H$ is any ball such that $v_{0} \in B(0, r)$ then there exists $t_{0}=t_{0}(r)$ such that (3.2) holds. This concludes the proof.

LEMma 3.2. There exists a bounded and absorbing set $V$ for the family of semiprocesses $\left\{U_{\sigma}(t, \tau)\right\}_{t \geq \tau \geq 0}, \sigma \in \Sigma$.

Proof. We multiply $(1.1)_{1}$ by $\operatorname{div} \mathbb{T}(v, p)$ and integrate over $\Omega$ to obtain

$$
\begin{array}{r}
\int_{\Omega} v_{, t} \cdot \operatorname{div} \mathbb{T}(v, p) d x-\int_{\Omega}|\operatorname{div} \mathbb{T}(v, p)|^{2} d x+\int_{\Omega} v \cdot \nabla v \cdot \operatorname{div} \mathbb{T}(v, p) d x \\
=\int_{\Omega} f \cdot \operatorname{div} \mathbb{T}(v, p) d x .
\end{array}
$$

According to the definition of $\mathbb{T}(v, p)$ we have

$$
\begin{aligned}
I_{1} & =\int_{\Omega} v_{, t} \cdot \operatorname{div}(\nu \mathbb{D}(v)-p I) d x \\
& =\nu \int_{\Omega} v_{, t} \operatorname{div} \mathbb{D}(v) d x-\int_{\Omega} v_{, t} \cdot \nabla p d x=I_{2}+I_{3} .
\end{aligned}
$$

From the Stokes theorem it follows that

$$
\begin{aligned}
I_{2} & =\nu \int_{\Omega} \operatorname{div}\left(v_{, t} \cdot \mathbb{D}(v)\right) d x-\nu \int_{\Omega} \nabla v, t \mathbb{D}(v) d x \\
& =\nu \int_{S} v_{, t} \cdot \mathbb{D}(v) \cdot \bar{n} d S-\frac{\nu}{4} \frac{d}{d t} \int_{\Omega}|D(v)|^{2} d x .
\end{aligned}
$$


Next we estimate the boundary integral. Using the boundary conditions we have

$$
\begin{aligned}
\int_{S} v_{, t} \cdot \mathbb{D}(v) \cdot & \bar{n} d S=\int_{S}\left((v \cdot \bar{n})_{, t} \bar{n}+\left(v \cdot \bar{\tau}_{\alpha}\right)_{, t} \bar{\tau}_{\alpha}\right) \cdot \mathbb{D}(v) \cdot \bar{n} d S \\
= & \int_{S_{1}}\left((v \cdot \bar{n})_{, t} \bar{n}+\left(v \cdot \bar{\tau}_{\alpha}\right)_{, t} \bar{\tau}_{\alpha}\right) \cdot \mathbb{D}(v) \cdot \bar{n} d S_{1} \\
& +\int_{S_{2}}\left((v \cdot \bar{n})_{, t} \bar{n}+\left(v \cdot \bar{\tau}_{\alpha}\right)_{, t} \bar{\tau}_{\alpha}\right) \cdot \mathbb{D}(v) \cdot \bar{n} d S_{2} \\
= & -\gamma \int_{S_{1}}\left(v \cdot \bar{\tau}_{\alpha}\right)_{, t} v \cdot \bar{\tau}_{\alpha} d S_{1}+\int_{S_{2}}(v \cdot \bar{n})_{, t} \bar{n} \cdot \mathbb{D}(v) \cdot \bar{n} d S_{2} \\
= & \frac{-\gamma}{2} \frac{d}{d t} \int_{S_{1}}\left(v \cdot \bar{\tau}_{\alpha}\right)^{2} d S_{1}+\int_{S_{2}} d_{, t} v_{3, x_{3}} d S_{2} \\
\leq & \frac{-\gamma}{2} \frac{d}{d t} \int_{S_{1}}\left(v \cdot \bar{\tau}_{\alpha}\right)^{2} d S_{1}+c\left|d_{, t}\right|_{2, S_{2}}^{2}+\varepsilon\|v\|_{2, \Omega}^{2} .
\end{aligned}
$$

Integrating by parts and using (2.5) we obtain

$$
\begin{aligned}
I_{3} & =\int_{S} v_{, t} \cdot \bar{n} p d S=\int_{S_{2}} d_{, t} p d S_{2} \leq\left(\left|d_{, t}\right|_{2, S_{2}}^{2}+\|p\|_{1, \Omega}^{2}\right) \\
& \leq c\left(\left|d_{, t}\right|_{2, S_{2}}^{2}+|f|_{2, \Omega}^{2}+|v|_{2, \Omega}^{2}+\|d\|_{3 / 2,2, S_{2}}^{2}+\left\|v \cdot \bar{\tau}_{\alpha}\right\|_{1 / 2,2, S_{1}}^{2}\right) .
\end{aligned}
$$

Finally, we get

$$
\begin{aligned}
I_{1} \leq & -\frac{\nu}{4} \frac{d}{d t} \int_{\Omega}|\mathbb{D}(v)|^{2} d x-\frac{\nu \gamma}{2} \frac{d}{d t} \int_{S_{1}}\left(v \cdot \bar{\tau}_{\alpha}\right)^{2} d S_{1}+\varepsilon\|v\|_{2, \Omega}^{2} \\
& +c\left(\left|d_{, t}\right|_{2, S_{2}}^{2}+|f|_{2, \Omega}^{2}+|v|_{2, \Omega}^{2}+\|d\|_{3 / 2,2, S_{2}}^{2}+\left\|v \cdot \bar{\tau}_{\alpha}\right\|_{1 / 2,2, S_{1}}^{2}\right) .
\end{aligned}
$$

Next we estimate the integral $\int_{\Omega} v \cdot \nabla v \cdot \operatorname{div} \mathbb{T}(v, p) d x$. From Lemma 2.3 we get

$$
\begin{aligned}
\int_{\Omega} v \cdot \nabla v \cdot \operatorname{div} \mathbb{T}(v, p) d x \leq \nu|v|_{6, \Omega}|\nabla v|_{3, \Omega}\left(|\operatorname{div} \mathbb{D}(v)|_{2, \Omega}+|\nabla p|_{2, \Omega}\right) \\
\leq c|v|_{6, \Omega}|\nabla v|_{3, \Omega}\left(|\operatorname{div} \mathbb{D}(v)|_{2, \Omega}+\|d\|_{3 / 2,2, S_{2}}+|f|_{2, \Omega}+|v|_{2, \Omega}\right. \\
\left.\quad+\gamma\left\|v \cdot \tau_{\alpha}\right\|_{1 / 2,2, S_{1}}\right)=I_{4}
\end{aligned}
$$

Using the interpolation inequality, the Young inequality and Lemma 2.2 yields 


$$
\begin{aligned}
I_{4} \leq & c|v|_{6, \Omega}|\nabla v|_{2, \Omega}^{1 / 2}|\nabla v|_{6, \Omega}^{1 / 2}\left(|\operatorname{div} \mathbb{D}(v)|_{2, \Omega}+\|d\|_{3 / 2,2, S_{2}}\right. \\
& \left.+|f|_{2, \Omega}+|v|_{2, \Omega}+\gamma\left\|v \cdot \bar{\tau}_{\alpha}\right\|_{1 / 2,2, S_{1}}\right) \\
\leq & c\|v\|_{1, \Omega}^{3 / 2}\|v\|_{2, \Omega}^{1 / 2}\left(|\operatorname{div} \mathbb{D}(v)|_{2, \Omega}+\|d\|_{3 / 2,2, S_{2}}+|f|_{2, \Omega}+|v|_{2, \Omega}\right. \\
& \left.+\gamma\left\|v \cdot \bar{\tau}_{\alpha}\right\|_{1 / 2,2, S_{1}}\right) \\
\leq & c\|v\|_{1, \Omega}^{3 / 2}\left(|\operatorname{div} \mathbb{D}(v)|_{2, \Omega}^{3 / 2}+\|d\|_{3 / 2,2, S_{2}}^{3 / 2}+|f|_{2, \Omega}^{3 / 2}\right. \\
& \left.+|v|_{2, \Omega}^{3 / 2}+\left(\gamma\left\|v \cdot \bar{\tau}_{\alpha}\right\|_{1 / 2,2, S_{1}}\right)^{3 / 2}\right) \\
\leq & c\left(\frac{1}{\varepsilon}\|v\|_{1, \Omega}^{6}+\varepsilon|\operatorname{div} \mathbb{D}(v)|_{2, \Omega}^{2}\right. \\
& \left.+\varepsilon|f|_{2, \Omega}^{2}+\varepsilon|v|_{2, \Omega}^{2}+\varepsilon\|d\|_{3 / 2,2, S_{2}}^{2}+\varepsilon \gamma^{2}\left\|v \cdot \bar{\tau}_{\alpha}\right\|_{1 / 2,2, S_{1}}^{2}\right)
\end{aligned}
$$

Hence we obtain from (3.3) the inequality

$$
\begin{aligned}
\frac{\nu}{4} \frac{d}{d t} \int_{\Omega}|\mathbb{D}(v)|^{2} d x+ & \frac{\nu \gamma}{2} \frac{d}{d t} \int_{S_{1}}\left(v \cdot \bar{\tau}_{\alpha}\right)^{2} d S_{1}+\int_{\Omega}|\operatorname{div} \mathbb{T}(v, p)|^{2} d x \\
\leq & \left|\int_{\Omega} f \cdot \operatorname{div} \mathbb{T}(v, p) d x\right|+\varepsilon\|v\|_{2, \Omega}^{2}+c\left|d_{, t}\right|_{2, S_{2}}^{2} \\
& +\frac{c}{\varepsilon}\left(\|v\|_{1, \Omega}^{6}+\|d\|_{3 / 2,2, S_{2}}^{2}+|f|_{2, \Omega}^{2}+|v|_{2, \Omega}^{2}\right. \\
& \left.+\left|d_{, t}\right|_{2, S_{2}}^{2}+\gamma^{2}\left\|v \cdot \bar{\tau}_{\alpha}\right\|_{1 / 2,2, S_{1}}^{2}\right)+\varepsilon|\operatorname{div} \mathbb{D}(v)|_{2, \Omega}^{2}
\end{aligned}
$$

Multiplying by $-\nu / 4$, using the Hölder inequality on the right hand side and observing that

$$
|\operatorname{div} \mathbb{D}(v)|_{2, \Omega} \leq c\left(|\operatorname{div} \mathbb{T}(v, p)|_{2, \Omega}+\|d\|_{3 / 2,2, S_{2}}+\gamma\left\|v \cdot \bar{\tau}_{\alpha}\right\|_{1 / 2,2, S_{1}}\right),
$$

we get

$$
\begin{aligned}
& \frac{d}{d t}|\mathbb{D}(v)|_{2, \Omega}^{2}+2 \gamma \frac{d}{d t}\left|v \cdot \bar{\tau}_{\alpha}\right|_{2, S_{1}}^{2}+|\operatorname{div} \mathbb{D}(v)|_{2, \Omega}^{2} \\
& \leq \varepsilon\|v\|_{2, \Omega}^{2}+\frac{c}{\varepsilon}\left(\left|d_{, t}\right|_{2, S_{2}}^{2}+\|d\|_{3 / 2,2, S_{2}}^{2}+\gamma^{2}\left\|v \cdot \bar{\tau}_{\alpha}\right\|_{1 / 2,2, S_{1}}^{2}+|v|_{2, \Omega}^{2}\right. \\
& \left.\quad+\|v\|_{1, \Omega}^{6}+|f|_{2, \Omega}^{2}\right) .
\end{aligned}
$$

Next using in (3.5) the inequalities

$$
|\mathbb{D}(v)|_{2, \Omega}+\|v\|_{2, \Omega} \leq c\left(|\operatorname{div} \mathbb{D}(v)|_{2, \Omega}+\gamma\left\|v \cdot \bar{\tau}_{\alpha}\right\|_{1 / 2,2, S_{1}}+\|d\|_{3 / 2,2, S_{2}}\right)
$$

and $\left\|v \cdot \bar{\tau}_{\alpha}\right\|_{1 / 2,2, S_{1}} \leq c\|v\|_{1, \Omega}$ we obtain

$$
\begin{aligned}
& \frac{d}{d t}|\mathbb{D}(v)|_{2, \Omega}^{2}+2 \gamma \frac{d}{d t}\left|v \cdot \bar{\tau}_{\alpha}\right|_{2, S_{1}}^{2}+|\mathbb{D}(v)|_{2, \Omega}^{2} \\
& \quad \leq c\left(|d,|_{2, S_{2}}^{2}+\|d\|_{3 / 2,2, S_{2}}^{2}+\gamma^{2}\|v\|_{1, \Omega}^{2}+\|v\|_{1, \Omega}^{6}+|f|_{2, \Omega}^{2}\right) \equiv J(t) .
\end{aligned}
$$


From (2.8) we get

$$
\int_{k T}^{(k+1) T}|\mathbb{D}(v(x, s))|_{2, \Omega}^{2} d s \leq a_{3}, \quad \int_{k T}^{(k+1) T} J(s) d s \leq a_{2} .
$$

Applying now Lemma 2.4 yields

$$
|\mathbb{D}(v)|_{2, \Omega}^{2} \leq a_{3} / T+a_{2},
$$

where $t \geq T$.

Finally, using the Korn inequality we obtain from (3.6)

$$
\frac{1}{c_{1}}\|v(t)\|_{1, \Omega}^{2} \leq \frac{a_{3}}{T}+a_{2} \quad \text { for } t \geq T,
$$

where $c_{1}$ is the constant from the Korn inequality.

We see that

$$
v(t) \in B\left(0, \rho_{2}\right) \quad \text { for all } t \geq t_{0},
$$

where $B\left(0, \rho_{2}\right)$ is the ball in $V$ centered at 0 with radius $\rho_{2}>c_{1}\left(a_{3} / T+a_{2}\right)$. If $B(0, r) \subset H$ is any ball such that $v_{0} \in B(0, r)$ then there exists $t_{0}=t_{0}(r)$ such that (3.5) holds. This concludes the proof.

Proof of Theorem 3.2. We take $\rho=\max \left\{\rho_{1}, \rho_{2}\right\}$. Then due to Lemmas 3.1 and 3.2 there exists an absorbing set $B(0, \rho)$ which is bounded in $V$ and bounded and compact in $H$. From Theorem 3.1 we conclude the proof.

4. Convergence to stationary solutions. First we examine the stationary problem (1.1)

$$
\begin{array}{ll}
v_{\infty} \cdot \nabla v_{\infty}-\operatorname{div} \mathbb{T}\left(v_{\infty}, p_{\infty}\right)=f_{\infty} & \text { in } \Omega, \\
\operatorname{div} v_{\infty}=0 & \text { in } \Omega, \\
v_{\infty} \cdot \bar{n}=0 & \text { on } S_{1}, \\
\nu \bar{n} \cdot \mathbb{D}\left(v_{\infty}\right) \cdot \bar{\tau}_{\alpha}+\gamma v_{\infty} \cdot \bar{\tau}_{\alpha}=0, \quad \alpha=1,2, & \text { on } S_{1}, \\
v_{\infty} \cdot \bar{n}=d_{\infty} & \text { on } S_{2}, \\
\bar{n} \cdot \mathbb{D}\left(v_{\infty}\right) \cdot \bar{\tau}_{\alpha}=0, \quad \alpha=1,2, & \text { on } S_{2} .
\end{array}
$$

Let us introduce an extension $\alpha=\alpha(x) \in \mathbb{R}$ such that

$$
\left.\alpha\right|_{S_{2}(-a)}=d_{1 \infty},\left.\quad \alpha\right|_{S_{2}(a)}=d_{2 \infty} .
$$

Then we introduce the vector $b=(0,0, \alpha)$. Define $u=v_{\infty}-b$. Then

$$
\begin{array}{ll}
\operatorname{div} u=-\operatorname{div} b & \text { in } \Omega, \\
u \cdot \bar{n}=0 & \text { on } S .
\end{array}
$$


Hence we define a function $\varphi$ to be the solution to the problem

$$
\begin{array}{ll}
\Delta \varphi=-\operatorname{div} b & \text { in } \Omega, \\
\nabla \varphi \cdot \bar{n}=0 & \text { on } S, \\
\int_{\Omega} \varphi d x=0 . &
\end{array}
$$

Next, we introduce the new function

$$
w=u-\nabla \varphi=v_{\infty}-(b+\nabla \varphi) \equiv v_{\infty}-\delta,
$$

which is the solution to the problem

$$
\begin{array}{ll}
(w+\delta) \cdot \nabla(w+\delta)-\operatorname{div} \mathbb{T}(w+\delta, p)=f_{\infty} & \text { in } \Omega, \\
\operatorname{div} w=0 & \text { in } \Omega, \\
w \cdot \bar{n}=0 & \text { on } S_{1}, \\
\nu \bar{n} \cdot \mathbb{D}(w+\delta) \cdot \bar{\tau}_{\alpha}+\gamma(w+\delta) \cdot \bar{\tau}_{\alpha}=0, \quad \alpha=1,2, & \text { on } S_{1}, \\
w \cdot \bar{n}=0 & \text { on } S_{2}, \\
\bar{n} \cdot \mathbb{D}(w+\delta) \cdot \bar{\tau}_{\alpha}=0, \quad \alpha=1,2, & \text { on } S_{2} .
\end{array}
$$

Multiplying (4.3) by $w$, integrating the result over $\Omega$ and next by parts we obtain the inequality

$$
\begin{aligned}
\nu \int_{\Omega}|\mathbb{D}(w)|^{2} d x+\gamma \int_{S_{1}} \mid & \left.w \cdot \bar{\tau}_{\alpha}\right|^{2} d S_{1} \\
\leq & \varepsilon\|w\|_{H^{1}(\Omega)}^{2}+\frac{1}{\varepsilon}\left(\nu\|\delta\|_{H^{1}(\Omega)}^{2}+\left\|f_{\infty}\right\|_{L_{6 / 5}(\Omega)}^{2}\right) \\
& \quad+\left|\int_{\Omega}(w+\delta) \cdot \nabla(w+\delta) w d x\right| .
\end{aligned}
$$

Now we examine the last term on the r.h.s. of (4.4):

$$
\begin{aligned}
& \left|\int_{\Omega}(w+\delta) \cdot \nabla(w+\delta) w d x\right| \\
\leq & \int_{\Omega}|w \cdot \nabla \delta w| d x+\int_{\Omega}|\delta \cdot \nabla w w| d x+\int_{\Omega}|\delta \cdot \nabla \delta w| d x \\
\leq & \varepsilon_{1}\|w\|_{L_{6}(\Omega)}^{2}+\frac{1}{\varepsilon_{1}}\|\nabla \delta\|_{L_{3 / 2}(\Omega)}^{2}\|w\|_{L_{6}(\Omega)}^{2} \varepsilon_{2}\|\nabla w\|_{L_{2}(\Omega)}^{2} \\
& +\frac{1}{\varepsilon_{2}}\|\delta\|_{L_{3}(\Omega)}^{2}\|w\|_{L_{6}(\Omega)}^{2}+\varepsilon_{3}\|w\|_{L_{6}(\Omega)}^{2}+\frac{1}{\varepsilon_{3}}\|\nabla \delta\|_{L_{3 / 2}(\Omega)}^{2}\|\delta\|_{L_{6}(\Omega)}^{2} \equiv I .
\end{aligned}
$$

Using the Korn inequality $\|w\|_{H^{1}(\Omega)} \leq c_{1}\|\mathbb{D}(w)\|_{L_{2}(\Omega)}^{2}$ we obtain from (4.4), (4.5), for sufficiently small $\varepsilon, \varepsilon_{1}, \varepsilon_{2}, \varepsilon_{3}$, the inequality 


$$
\begin{aligned}
& \frac{\nu}{c_{1}}\|w\|_{H^{1}(\Omega)}^{2}+\gamma \int_{S_{1}}\left|w \cdot \bar{\tau}_{\alpha}\right|^{2} d S_{1} \\
\leq & c_{2}\left(\|\delta\|_{H^{1}(\Omega)}^{2}+\left\|f_{\infty}\right\|_{L_{6 / 5}(\Omega)}^{2}\right)+c_{3}\|\delta\|_{H^{1}(\Omega)}^{2}\|w\|_{H^{1}(\Omega)}^{2}+c\|\delta\|_{H^{1}(\Omega)}^{4} .
\end{aligned}
$$

Assuming

$$
c_{3}\|\delta\|_{H^{1}(\Omega)}^{2} \leq \frac{1}{2} \frac{\nu}{c_{1}},
$$

we obtain from (4.6) the inequality

$$
\begin{aligned}
\frac{1}{2} \frac{\nu}{c_{1}}\|w\|_{H^{1}(\Omega)}^{2}+\gamma \| w & \cdot \bar{\tau}_{\alpha} \|_{L_{2}\left(S_{1}\right)}^{2} \\
& \leq c_{2}\left(\|\delta\|_{H^{1}(\Omega)}^{2}+\left\|f_{\infty}\right\|_{L_{6 / 5}(\Omega)}^{2}\right)+c\|\delta\|_{H^{1}(\Omega)}^{4} .
\end{aligned}
$$

Next, (4.2) and (4.7) imply

$$
\frac{1}{2} \frac{\nu}{c_{1}}\left\|v_{\infty}\right\|_{H^{1}(\Omega)}^{2} \leq c_{4}\|\delta\|_{H^{1}(\Omega)}^{2}+c_{2}\left\|f_{\infty}\right\|_{L_{6 / 5}(\Omega)}^{2}+c\|\delta\|_{H^{1}(\Omega)}^{4} .
$$

where $c_{4}=c_{2}+\frac{1}{2} \frac{\nu}{c_{1}}$. Let $V=v(t)-v_{\infty}, P=p(t)-p_{\infty}$. Then $(V, P)$ satisfies the system of equations

$$
\begin{array}{ll}
\begin{array}{ll}
V_{t}-\operatorname{div} \mathbb{T}(V, P)=V \cdot \nabla V+V \cdot \nabla v_{\infty} & \\
& +v_{\infty} \cdot \nabla V+f-f_{\infty}
\end{array} & \text { in } \Omega^{T}, \\
\operatorname{div} V=0 & \text { in } \Omega^{T}, \\
V \cdot \bar{n}=0 & \text { on } S_{1}^{T}, \\
\nu \bar{n} \cdot \mathbb{D}(V) \cdot \bar{\tau}_{\alpha}+\gamma V \cdot \bar{\tau}_{\alpha}=0, \quad \alpha=1,2 & \text { on } S_{1}^{T}, \\
v \cdot \bar{n}=d(t)-d_{\infty} & \text { on } S_{2}^{T}, \\
\bar{n} \cdot \mathbb{D}(V) \cdot \bar{\tau}_{\alpha}=0, \quad \alpha=1,2 & \text { on } S_{2}^{T}, \\
\left.V\right|_{t=0}=v(0)-v_{\infty} \equiv V(0) & \text { in } \Omega .
\end{array}
$$

To obtain an energy type estimate for solutions to problem (4.9) we introduce the function

$$
\left.\alpha_{1}\right|_{S_{2}(-a)}=d_{1}-d_{1 \infty},\left.\quad \alpha_{1}\right|_{S_{2}(a)}=d_{2}-d_{2 \infty} .
$$

Then we define $u_{1}$ by

$$
\begin{aligned}
& \operatorname{div} u_{1}=-\operatorname{div} b_{1}, \\
& \left.u_{1} \cdot \bar{n}\right|_{S}=0,
\end{aligned}
$$

where $b_{1}=\left(0,0, \alpha_{1}\right)$. Next we introduce the function $\varphi_{1}$ by

$$
\begin{array}{ll}
\Delta \varphi_{1}=-\operatorname{div} b_{1} & \text { in } \Omega, \\
\bar{n} \cdot \nabla \varphi_{1}=0 & \text { on } S, \\
\int_{\Omega} \varphi_{1} d x=0 . &
\end{array}
$$


Finally, the function

$$
W=u_{1}-\nabla \varphi_{1}=V-\left(b_{1}+\nabla \varphi_{1}\right) \equiv V-\delta_{1}
$$

and $P$ solve the problem

$$
\begin{aligned}
& W_{, t}-\operatorname{div} \mathbb{T}(W, P)=-\delta_{1, t}+\nu \operatorname{div} \mathbb{D}\left(\delta_{1}\right) \\
& +\left(W+\delta_{1}\right) \cdot \nabla\left(W+\delta_{1}\right)+\left(W+\delta_{1}\right) \cdot \nabla v_{\infty} \\
& +v_{\infty} \cdot \nabla\left(W+\delta_{1}\right)+f-f_{\infty} \quad \text { in } \Omega^{T}, \\
& \operatorname{div} W=0 \quad \text { in } \Omega^{T}, \\
& W \cdot \bar{n}=0 \quad \text { on } S^{T}, \\
& \nu \bar{n} \cdot \mathbb{D}\left(W+\delta_{1}\right) \cdot \bar{\tau}_{\alpha}+\gamma\left(W+\delta_{1}\right) \cdot \bar{\tau}_{\alpha}=0, \quad \alpha=1,2, \quad \text { on } S_{1}^{T}, \\
& \bar{n} \cdot \mathbb{D}\left(W+\delta_{1}\right) \cdot \bar{\tau}_{\alpha}=0, \quad \alpha=1,2, \quad \text { on } S_{2}^{T}, \\
& \left.W\right|_{t=0}=V(0)-\delta_{1}(0) .
\end{aligned}
$$

Multiplying (4.14) ${ }_{1}$ by $W$ and integrating over $\Omega$ yields

$$
\begin{aligned}
& \frac{1}{2} \frac{d}{d t}\|W\|_{L_{2}(\Omega)}^{2}-\int_{\Omega} \operatorname{div} \mathbb{T}(W, P) \cdot W d x \\
& =\int_{\Omega}\left(-\delta_{1, t}+\nu \operatorname{div} \mathbb{D}\left(\delta_{1}\right)\right) \cdot W d x \\
& \quad+\int_{\Omega}\left(W+\delta_{1}\right) \cdot \nabla\left(W+\delta_{1}\right) \cdot W d x+\int_{\Omega}\left(W+\delta_{1}\right) \cdot \nabla v_{\infty} \cdot W d x \\
& \quad+\int_{\Omega} v_{\infty} \cdot \nabla\left(W+\delta_{1}\right) \cdot W d x+\int_{\Omega}\left(f-f_{\infty}\right) \cdot W d x \\
& \equiv \sum_{i=1}^{5} I_{i} .
\end{aligned}
$$

The second term on the l.h.s. equals

$$
-\int_{S} \bar{n} \cdot \mathbb{T}(W, P) \cdot W d S+\nu \int_{\Omega}|\mathbb{D}(W)|^{2} d x,
$$

where the boundary term takes the form

$$
\begin{aligned}
& -\int_{S_{1}}\left[-\nu \bar{n} \cdot \mathbb{D}\left(\delta_{1}\right) \cdot \bar{\tau}_{\alpha}-\gamma\left(W+\delta_{1}\right) \cdot \bar{\tau}_{\alpha}\right] W \cdot \bar{\tau}_{\alpha} d S_{1} \\
& -\int_{S_{2}} \nu \bar{n} \cdot \mathbb{D}\left(\delta_{1}\right) \cdot \bar{\tau}_{\alpha} W \cdot \bar{\tau}_{\alpha} d S_{2} .
\end{aligned}
$$

Hence

$$
-\int_{S} \bar{n} \cdot \mathbb{T}(W, P) \cdot W d S \geq \gamma \int_{S_{1}}\left|W \cdot \bar{\tau}_{\alpha}\right|^{2} d S_{1}+I,
$$


where

$$
|I| \leq \varepsilon\|W\|_{H^{1}(\Omega)}^{2}+(c / \varepsilon)\left\|\delta_{1}\right\|_{H^{2}(\Omega)}^{2} .
$$

Now we estimate the terms on the r.h.s. of (4.15):

$$
\begin{aligned}
I_{1} & \leq \varepsilon\|W\|_{H^{1}(\Omega)}^{2}+(c / \varepsilon)\left(\left\|\delta_{1, t}\right\|_{L_{2}(\Omega)}^{2}+\left\|\delta_{1}\right\|_{H^{2}(\Omega)}^{2}\right), \\
I_{2} & =\int_{\Omega}\left[W \cdot \nabla \delta_{1} \cdot W+\delta_{1} \cdot \nabla\left(W+\delta_{1}\right) \cdot W\right] d x \\
& \leq \varepsilon\|W\|_{H^{1}(\Omega)}^{2}+(c / \varepsilon)\left(\left\|\delta_{1}\right\|_{L_{\infty}(\Omega)}^{2}\|W\|_{L_{2}(\Omega)}^{2}+\left\|\delta_{1}\right\|_{H^{1}(\Omega)}^{4}\right), \\
I_{3} & \leq \varepsilon\|W\|_{L_{6}(\Omega)}^{2}+(c / \varepsilon)\left\|\nabla v_{\infty}\right\|_{L_{3}(\Omega)}^{2}\left(\|W\|_{L_{2}(\Omega)}^{2}+\left\|\delta_{1}\right\|_{L_{2}(\Omega)}^{2}\right), \\
I_{4} & \leq \varepsilon\|\nabla W\|_{L_{2}(\Omega)}^{2}+(c / \varepsilon)\left\|v_{\infty}\right\|_{L_{\infty}(\Omega)}^{2}\left(\|W\|_{L_{2}(\Omega)}^{2}+\left\|\delta_{1}\right\|_{H^{1}(\Omega)}^{2}\right), \\
I_{5} & \leq \varepsilon\|W\|_{L_{6}(\Omega)}^{2}+(c / \varepsilon)\left\|f-f_{\infty}\right\|_{L_{6 / 5}(\Omega)}^{2} .
\end{aligned}
$$

Using the Korn inequality and assuming that $\varepsilon$ is sufficiently small we obtain the inequality

$$
\begin{aligned}
& \frac{d}{d t}\|W\|_{L_{2}(\Omega)}^{2}+\nu\|W\|_{H^{1}(\Omega)}^{2}+\gamma\left\|W \cdot \bar{\tau}_{\alpha}\right\|_{L_{2}\left(S_{1}\right)}^{2} \\
& \leq \frac{6}{\nu}\left(\left\|\delta_{1}\right\|_{L_{\infty}(\Omega)}^{2}+\left\|v_{\infty}\right\|_{W_{4}^{1}(\Omega)}^{2}\right)\|W\|_{L_{2}(\Omega)}^{2} \\
& \quad+c\left(\left\|\delta_{1}\right\|_{H^{2}(\Omega)}^{2}+\left\|\delta_{1, t}\right\|_{L_{2}(\Omega)}^{2}+\left\|\delta_{1}\right\|_{H^{1}(\Omega)}^{4}+\left\|f-f_{\infty}\right\|_{L_{6 / 5}(\Omega)}^{2}\right) .
\end{aligned}
$$

Continuing, we get

$$
\begin{aligned}
\frac{d}{d t}\left(\|W\|_{L_{2}(\Omega)}^{2} e^{\nu t-\frac{6}{\nu} \int_{0}^{t}\left(\left\|\delta_{1}\right\|_{L_{\infty}(\Omega)}^{2}+\left\|v_{\infty}\right\|_{W_{4}^{1}(\Omega)}^{2}\right) d t^{\prime}}\right) \\
\leq c\left(\left\|\delta_{1}\right\|_{H^{2}(\Omega)}^{2}+\left\|\delta_{1, t}\right\|_{L_{2}(\Omega)}^{2}+\left\|\delta_{1}\right\|_{H^{1}(\Omega)}^{4}\right. \\
\left.\quad+\left\|f-f_{\infty}\right\|_{L_{6 / 5}(\Omega)}^{2}\right) e^{\nu t-\frac{6}{\nu} \int_{0}^{t}\left(\left\|\delta_{1}\right\|_{L_{\infty}(\Omega)}^{2}+\left\|v_{\infty}\right\|_{W_{4}^{1}(\Omega)}^{2}\right) d t^{\prime}} .
\end{aligned}
$$

Integrating with respect to time yields

$$
\begin{aligned}
\| W(t) & \|_{L_{2}(\Omega)}^{2} \leq e^{-\nu t+\frac{6}{\nu} \int_{0}^{t}\left(\left\|\delta_{1}\right\|_{L_{\infty}(\Omega)}^{2}+\left\|v_{\infty}\right\|_{W_{4}^{1}(\Omega)}^{2}\right) d t^{\prime}} \\
& \cdot c \int_{0}^{t}\left(\left\|\delta_{1}\right\|_{H^{2}(\Omega)}^{2}+\left\|\delta_{1, t}\right\|_{L_{2}(\Omega)}^{2}+\left\|\delta_{1}\right\|_{H^{1}(\Omega)}^{4}+\left\|f-f_{\infty}\right\|_{L_{6 / 5}(\Omega)}^{2}\right) \\
& \cdot e^{\nu t^{\prime}-\frac{6}{\nu} t_{0}^{t^{\prime}}\left(\left\|\delta_{1}\right\|_{L_{\infty}(\Omega)}^{2}+\left\|v_{\infty}\right\|_{W_{4}^{1}(\Omega)}^{2}\right) d t^{\prime \prime}} d t^{\prime} \\
& +e^{-\nu t+\frac{6}{\nu} \int_{0}^{t}\left(\left\|\delta_{1}\right\|_{L_{\infty}(\Omega)}^{2}+\left\|v_{\infty}\right\|_{W_{4}^{1}(\Omega)}^{2}\right) d t^{\prime}}\|W(0)\|_{L_{2}(\Omega)}^{2} .
\end{aligned}
$$


Assuming that

$$
-\nu+\frac{6}{\nu}\left\|v_{\infty}\right\|_{W_{4}^{1}(\Omega)}^{2}+\frac{6}{\nu} \int_{0}^{t}\left\|\delta_{1}\right\|_{L_{\infty}(\Omega)}^{2} d t^{\prime} \leq-\frac{\nu}{2}
$$

and the decay

$$
\begin{aligned}
\left\|\delta_{1}(t)\right\|_{H^{2}(\Omega)}^{2}+ & \left\|\delta_{1, t}(t)\right\|_{L_{2}(\Omega)}^{2}+\left\|\delta_{1}(t)\right\|_{H^{1}(\Omega)}^{4}+\left\|f(t)-f_{\infty}\right\|_{L_{6 / 5}(\Omega)}^{2} \\
\leq & e^{-\delta t}\left(\left\|\delta_{1}(0)\right\|_{H^{2}(\Omega)}^{2}+\left\|\delta_{1, t}(0)\right\|_{L_{2}(\Omega)}^{2}+\left\|\delta_{1}(0)\right\|_{H^{1}(\Omega)}^{4}\right. \\
& \left.+\left\|f(0)-f_{\infty}\right\|_{L_{6 / 5}(\Omega)}^{2}\right)
\end{aligned}
$$

we obtain from (4.14) the inequality

$$
\begin{aligned}
\| W(t) & \|_{L_{2}(\Omega)}^{2} \leq c e^{-\delta t}\left(\left\|\delta_{1}(0)\right\|_{H^{2}(\Omega)}^{2}+\left\|\delta_{1, t}(0)\right\|_{L_{2}(\Omega)}^{2}\right. \\
& \left.+\left\|\delta_{1}(0)\right\|_{H^{1}(\Omega)}^{4}+\left\|f(0)-f_{\infty}\right\|_{L_{6 / 5}(\Omega)}^{2}\right)+e^{-\nu t / 2}\|W(0)\|_{L_{2}(\Omega)}^{2}
\end{aligned}
$$

Therefore we obtain

THEOREM 4.1. Let $f$ denote the external field force in the nonstationary problem (1.1), and $f_{\infty}$ the external field force in the stationary problem. Assume that $f_{\infty} \in L_{6 / 5}(\Omega)$. If

$$
\left\|f(t)-f_{\infty}\right\|_{L_{6 / 5(\Omega)}} \underset{t \rightarrow \infty}{\longrightarrow} 0 \quad \text { and } \quad\left\|d-d_{\infty}\right\|_{L_{2}\left(S_{2}\right)} \underset{t \rightarrow \infty}{\longrightarrow} 0
$$

then the solution $v(t)$ of problem (1.1) converges to the stationary solution $v_{\infty}$ of problem (1.1) and the estimate

$$
\begin{aligned}
\left\|v(t)-v_{\infty}\right\|_{L_{2}(\Omega)}^{2} \leq c e^{-\delta t}\left(\left\|\delta_{1}(0)\right\|_{H^{2}(\Omega)}^{2}+\left\|\delta_{1, t}(0)\right\|_{L_{2}(\Omega)}^{2}\right. \\
\left.\quad+\left\|\delta_{1}(0)\right\|_{H^{1}(\Omega)}^{4}+\left\|f(0)-f_{\infty}\right\|_{L_{6 / 5}(\Omega)}^{2}\right)+e^{-\nu t / 2}\left\|v(0)-v_{\infty}\right\|_{L_{2}(\Omega)}^{2}
\end{aligned}
$$

holds, where $\delta, \delta_{1}$ are given by (4.2) and (4.10).

Acknowledgments. The author thanks Prof. W. Zajączkowski for important discussions during the preparation of this paper.

This research was partially supported by the Polish KBN Grant 1/P03A/ $021 / 30$.

\section{References}

[1] V. Chepyzhov and M. Vishik, Attractors for Equations of Mathematical Physics, Amer. Math. Soc., RI, 2001.

[2] P. Kacprzyk, Global regular nonstationary flow for the Navier-Stokes equations in a cylindrical pipe, Appl. Math. (Warsaw) 34 (2007), 289-307.

[3] - Global existence for the inflow-outflow problem for the Navier-Stokes equations in a cylinder, ibid. 36 (2009), 195-212.

[4] T. Kobayashi and W. M. Zajączkowski, On global motion of a compressible barotropic viscous fluid with boundary slip conditions, ibid. 26 (1999), 159-194. 
[5] O. A. Ladyzhenskaya and V. A. Solonnikov, On some problems of vector analysis and generalized boundary value problems for the Navier-Stokes equations, Zap. Nauchn. Sem. LOMI 59 (1976), 81-116 (in Russian).

[6] O. A. Ladyzhenskaya, V. A. Solonnikov and N. N. Ural'tseva, Linear and Quasilinear Equations of Parabolic Type, Nauka, Moscow, 1967 (in Russian).

[7] B. Nowakowski and W. M. Zajączkowski, Global attractor for Navier-Stokes equations in cylindrical domains, Appl. Math. (Warsaw) 36 (2009), 183-194.

[8] - - - Global existence of solutions to Navier-Stokes equations in cylindrical domains, ibid. 36 (2009), 169-182.

[9] J. Rencławowicz and W. M. Zajączkowski, Large time regular solutions to the Navier-Stokes equations in cylindrical domains, Topol. Methods Nonlinear Anal. 32 (2008), 69-87.

[10] V. A. Solonnikov, On general boundary value problems for Douglis-Nirenberg elliptic systems, Trudy Mat. Inst. Steklov. 92 (1966), 233-297 (in Russian).

[11] R. Temam, Navier-Stokes Equations, North-Holland, 1979.

[12] -, Infinite-Dimensional Dynamical Systems in Mechanics and Physics, Appl. Math. Sci. 68, Springer 1997.

[13] M. Wiegner and W. M. Zajączkowski, On stability of axially symmetric solutions to Navier-Stokes equations in a cylindrical domain and with boundary slip conditions, in: Regularity and Other Aspects of the Navier-Stokes Equations, Banach Center Publ. 70, Inst. Math., Polish Acad. Sci., 2005, 251-278.

[14] W. M. Zajączkowski, Long time existence of regular solutions to Navier-Stokes equations in cylindrical domains under boundary slip conditions, Studia Math. 169 (2005), 243-285.

[15] - Global existence of axially symmetric solutions to Navier-Stokes equations with large angular component of velocity, Colloq. Math. 100 (2004), 243-263.

[16] - Global special regular solutions to the Navier-Stokes equations a cylindrical domain without the axis of symmetry, Topol. Methods Nonlinear Anal. 24 (2004), 60-105.

[17] - Global regular nonstationary flow for the Navier-Stokes equations in a cylindrical pipe, ibid. 26 (2005), 221-286.

Piotr Kacprzyk

Institute of Mathematics and Cryptology

Cybernetics Faculty

Military University of Technology

Kaliskiego 2

00-908 Warszawa, Poland

E-mail: pk_wat@wp.pl

Received 1.7.2008

and in final form 2\%.10.2009 
